Marek Balcerzak, Institute of Mathematics, Łódź Technical University, al. Politechniki 11, I-2, 90-924 Łódź, Poland and Faculty of Mathematics, University of Łódź, ul. Banacha 22, 90-238 Łódź, Poland. e-mail:

mbalce@krysia.uni.lodz.pl

Artur Wachowicz, Institute of Mathematics, Łódź Technical University, al. Politechniki 11, I-2, 90-924 Łódź, Poland.

\title{
SOME EXAMPLES OF MEAGER SETS IN BANACH SPACES
}

\author{
Abstract \\ We show that some sets in the spaces $c_{0} \times c_{0}, L^{1}[0,1] \times L^{1}[0,1]$ and \\ $C[0,1]$, that appear in analysis, are meager.
}

\section{Introduction}

We consider the following separable Banach spaces:

- the space $\mathbb{R}$ of real numbers;

- the space $c_{0}$ of sequences of reals convergent to zero, with the norm $\left\|\left(a_{n}\right)\right\|=\max _{n \in \mathbb{N}}\left|a_{n}\right|$

- the space $L^{1}[0,1]$ of Lebesgue integrable functions on $[0,1]$ (written briefly as $\left.L^{1}\right)$;

- the space $C[0,1]$ of real-valued continuous functions on $[0,1]$, with the sup norm.

We shall prove that some natural sets in the product $X \times Y$ (where $X, Y$ are the respective spaces from those listed above) are meager (i.e. of the first category). Consequently, the complements of these sets are comeager (or, residual) which mean that they describe some typical behaviour of objects in the space. Several strong theorems concerning typical properties belong to the classical real analysis. (See $[\mathrm{Br}]$ and $[\mathrm{Jo}]$.) One of the first unexpected

Key Words: Baire category, summable sequences, Lebesgue integrable functions, $\mathcal{I}$ approximate derivative

Mathematical Reviews subject classification: 54E52, 26A24, 40A05, 46A45

Received by the editors June 30, 2000 
results in that direction, due to Banach $[\mathrm{B}]$ and Mazurkiewicz $[\mathrm{M}]$, states that a typical continuous function on $[0,1]$ is nondifferentiable in any point. The first two of our theorems are connected with multiplying of two sequences in $c_{0}$ and of two functions in $L^{1}$. The idea of the proofs is elementary. We observe that the respective sets are $F_{\sigma}$ with empty interiors. The third theorem is deeper. Its proof uses a technique based on the Kuratowski-Ulam theorem and proposed by Kharazishvili in [Kh]. Our result describes the behaviour of a typical continuous function with respect to differentiability in the sense of the category approximate derivative, so it can be treated as the next result in the series following the classical Banach-Mazurkiewicz theorem.

\section{Some properties in $c_{0} \times c_{0}$ and $L^{1} \times L^{1}$}

Let $\mathbb{N}=\{1,2, \ldots\}$. Observe that two sequences $\left(a_{k}\right),\left(b_{k}\right)$ in $c_{0}$ can produce a sequence $\left(a_{k} b_{k}\right)$ for which the set of sums $\sum_{k=1}^{n} a_{k} b_{k}, n \in \mathbb{N}$, is unbounded. Put for instance $a_{k}=b_{k}=1 / \sqrt{k}, k \in \mathbb{N}$. We shall show that this behaviour is typical.

Theorem 1.1. The set

$$
E=\left\{\left(\left(a_{i}\right),\left(b_{i}\right)\right) \in c_{0} \times c_{0}:\left(\sum_{i=1}^{n} a_{i} b_{i}\right)_{n \in \mathbb{N}} \text { is bounded }\right\}
$$

is a meager set of type $F_{\sigma}$.

Proof. We have

$$
E=\bigcup_{m=1}^{\infty} \bigcap_{n=1}^{\infty} E_{m n}
$$

where

$$
E_{m n}=\left\{\left(\left(a_{k}\right),\left(b_{k}\right)\right) \in c_{0} \times c_{0}:\left|\sum_{k=1}^{n} a_{k} b_{k}\right| \leq m\right\} .
$$

Observe that each set $E_{m n}$ is closed. Hence $E$ is of type $F_{\sigma}$. Let $\left(a_{k}\right),\left(b_{k}\right) \in c_{0}$ and $\varepsilon>0$. Pick an $k_{0} \in \mathbb{N}$ such that $\left|a_{k}\right|<\varepsilon / 2$ and $\left|b_{k}\right|<\varepsilon / 2$ for each $k \geq k_{0}$. Let $k_{1}=\max \left\{k_{0}, 4 / \varepsilon^{2}\right\}$. Put $\tilde{a_{k}}=\tilde{b_{k}}=1 / \sqrt{k}$ for each $k \geq k_{1}$, and $\tilde{a_{k}}=a_{k}, \tilde{b_{k}}=b_{k}$, otherwise. Clearly, $\left\|\left(\tilde{a_{k}}\right)-\left(a_{k}\right)\right\|<\varepsilon,\left\|\left(\tilde{b_{k}}\right)-\left(b_{k}\right)\right\|<\varepsilon$, and

$$
\sum_{k=k_{0}}^{\infty} \tilde{a_{k}} \tilde{b_{k}}=\sum_{k=k_{0}}^{\infty} \frac{1}{k}=\infty
$$

Hence $\left(\left(\tilde{a_{k}}\right),\left(\tilde{b_{k}}\right)\right) \in\left(c_{0} \times c_{0}\right) \backslash E$. Consequently, $E$ is a boundary $F_{\sigma}$ set and therefore it is meager. 
Remark. A referee has observed that Theorem 1.1 resembles the classical Banach-Steinhaus theorem. However, for us it is unclear how to obtain our result as a simple corollary.

Now, we shall deal with multiplying of two Lebesgue integrable functions. First observe that two integrable functions $f, g$ on $[0,1]$ can produce a nonintegrable function $f g$. Namely, let for instance $f(x)=g(x)=1 / \sqrt{x}$ for $x \neq 0$, and $f(0)=g(0)=0$. We shall prove that this property is typical.

We need the following lemma:

Lemma 1.1. If $f_{k} \rightarrow f$ in $L^{1}$ then for each $c \geq 0$ we have $\min \left\{\left|f_{k}\right|, c\right\} \rightarrow$ $\min \{|f|, c\}$ in $L^{1}$.

Proof. We have

$$
\begin{gathered}
\int_{0}^{1}\left|\min \left\{\left|f_{k}\right|, c\right\}-\min \{|f|, c\}\right| \\
=\int_{\left\{|f| \leq c \wedge\left|f_{k}\right| \leq c\right\}}|| f_{k}|-| f||+\int_{\left\{|f| \leq c \wedge\left|f_{k}\right|>c\right\}}(c-|f|)+\int_{\left\{|f|>c \wedge\left|f_{k}\right| \leq c\right\}}\left(c-\left|f_{k}\right|\right) \\
\leq 3 \int_{0}^{1}|| f_{k}|-| f|| \leq 3 \int_{0}^{1}\left|f_{k}-f\right| \rightarrow 0 .
\end{gathered}
$$

Theorem 1.2. The set

$$
E=\left\{(f, g) \in L^{1} \times L^{1}: \int_{0}^{1}|f g|<\infty\right\}
$$

is meager of type $F_{\sigma}$.

Proof. We have

$$
E=\bigcup_{m=1}^{\infty} \bigcap_{n=1}^{\infty} E_{m n}
$$

where

$$
E_{m n}=\left\{(f, g) \in L^{1} \times L^{1}: \int_{0}^{1} \min \{|f|, n\} \min \{|g|, n\} \leq m\right\} .
$$

Each set $E_{m n}$ is closed. Indeed, fix $m, n \in \mathbb{N}$, and assume that $\left(f_{k}, g_{k}\right) \in E_{m n}$, $k \in \mathbb{N}$, and $\left(f_{k}, g_{k}\right) \rightarrow(f, g)$ in $L^{1} \times L^{1}$. For $\varphi \in L^{1}$ let $\varphi^{\star}=\min \{|\varphi|, n\}$. From Lemma 1.1 it follows that $\left(f_{k}^{\star}, g_{k}^{\star}\right) \rightarrow\left(f^{\star}, g^{\star}\right)$. We have

$$
\int_{0}^{1} f^{\star} g^{\star}=\int_{0}^{1}\left(f^{\star} g^{\star}-f_{k}^{\star} g^{\star}\right)+\int_{0}^{1}\left(f_{k}^{\star} g^{\star}-f_{k}^{\star} g_{k}^{\star}\right)+\int_{0}^{1} f_{k}^{\star} g_{k}^{\star}
$$




$$
\begin{aligned}
& \leq \int_{0}^{1} g^{\star}\left(f^{\star}-f_{k}^{\star}\right)+\int_{0}^{1} f_{k}^{\star}\left(g^{\star}-g_{k}^{\star}\right)+m \\
& \leq n\left\|f^{\star}-f_{k}^{\star}\right\|+n\left\|g^{\star}-g_{k}^{\star}\right\|+m \rightarrow m
\end{aligned}
$$

whenever $k \rightarrow \infty$. Thus $(f, g) \in E_{m n}$.

Now, let $f, g \in L^{1}$ and $\varepsilon>0$. By the absolute continuity of Lebesgue integral, pick an $a>0$ such that $\int_{0}^{a}|f|<\varepsilon / 2$ and $\int_{0}^{a}|g|<\varepsilon / 2$. Let $b=$ $\min \left\{a, \varepsilon^{2} / 16\right\}$. Define $\tilde{f}(x)=\tilde{g}(x)=1 / \sqrt{x}$ for each $x \in(0, b]$, and $\tilde{f}(x)=$ $f(x), \tilde{g}(x)=g(x)$ for the remaining $x$ 's in $[0,1]$. Then we have

$$
\|\tilde{f}-f\| \leq \int_{0}^{b} \frac{1}{\sqrt{x}}+\int_{0}^{b}|f|<\varepsilon \text { and similarly }\|\tilde{g}-g\|<\varepsilon .
$$

On the other hand

$$
\int_{0}^{b} \tilde{f} \tilde{g}=\int_{0}^{b} \frac{1}{x}=\infty
$$

Hence $(\tilde{f}, \tilde{g}) \in\left(L^{1} \times L^{1}\right) \backslash E$. Consequently, $E$ is a boundary $F_{\sigma}$ set and therefore it is meager.

\section{$2 \quad \mathcal{I}$-approximate differentiability of continuous functions}

Assume that $X$ and $Y$ are topological spaces and $Y$ possesses a countable $\pi$-base. For $E \subset X \times Y$ and $x \in X$ we let $E(x)=\{y \in Y:(x, y) \in E\}$. The Kuratowski-Ulam theorem [O] states that if $E$ is meager then the set $\{x \in X: E(x)$ is nonmeager $\}$ is meager. The converse is true provided $E$ possesses the Baire property. By a partial function from $X$ to $Y$ we mean a function, with values in $Y$, whose domain is contained in $X$.

In $[\mathrm{Kh}]$ Kharazishvili presented a useful version of the Kuratowski-Ulam theorem which can be formulated as follows.

Proposition 2.1. [Kh] Let $X$ be a topological space and let $Y, Z$ be topological vector spaces where $Y$ is additionally Polish and $Z$ has a countable base. Assume that a set $D \subset X \times Y$ and a mapping $\Phi: D \rightarrow Z$ possesses the Baire property. Assume also that for almost all (in the sense of category) points $x \in X$ the set $D(x)$ is a linear subspace of $Y$ and the mapping $\Phi(x, \cdot)$ is linear and discontinuous. Then $D$ is meager in $X \times Y$ and $\{x \in X: D(x)$ is nonmeager $\}$ is meager in $X$.

Our application of Proposition 2.1 concerns the $\mathcal{I}$-approximate derivative. Recall that Jarnik $[J]$ obtained a result dealing with continuous functions with no approximate derivative at any point, which is stronger than the classical 
Banach-Mazurkiewicz theorem. Kharazishvili [Kh], by the use of Proposition 2.1, proved a version of Jarnik's result stating that, for almost all (in the sense of category) functions in $C[0,1]$, a (finite) approximate derivative can exist for a meager set of points in $[0,1]$. Wilczyński in $[\mathrm{W}]$ introduced a category analog of a density point. Soon after that, a category version of an approximate derivative (called an $\mathcal{I}$-approximate derivative) appeared in [LW]. We shall present a category analog of the above-mentioned Kharazishvili theorem. This seems to be the first result of that kind concerning $\mathcal{I}$-approximate derivatives.

Let us start with definitions. Let $\mathcal{I}$ stand for the $\sigma$-ideal of all meager subsets of $\mathbb{R}$. We say $[\mathrm{W}]$ that a number $x \in \mathbb{R}$ is an $\mathcal{I}$-density point of a set $A \subset \mathbb{R}$ with the Baire property if each increasing sequence $\left(n_{k}\right)$ of positive integers has a subsequence $\left(n_{m_{k}}\right)$ such that the sequence of characteristic functions

$$
\chi_{(-1,1) \cap n_{m_{k}}(A-x)}(t)
$$

(where $n(A-x)=\{n(a-x): a \in A\})$ tends to $\chi_{(-1,1)}(t)$ for all points $t \in \mathbb{R}$ except for those that belong to a meager set. There exist several equivalent variants of the above definition that are useful in various situations. One of the possible formulations is the following

Lemma 2.1. (See $[C L O$, Th. 2.2.2(vii)].) A number $x \in \mathbb{R}$ is an $\mathcal{I}$-density point of a set $A \subset \mathbb{R}$ with the Baire property if and only if

$(\forall(a, b) \subset(-1,1))(\exists k, m)(\forall n>m)(\exists(c, d) \subset(a, b))((c, d) \subset n(A-x) \wedge|d-c| \geq 1 / k)$

where $a, b, c, d$, are rationals and $k, m, n$ are positive integers.

If, in the above definition and Lemma 2.1 , we replace $(-1,1)$ by $(0,1)$ (respectively, by $(-1,0)$ ), we get a notion of the right-hand $\mathcal{I}$-density point of $A$ (left-hand $\mathcal{I}$-density point of $A$ ).

Assume now that a function $f:[0,1] \rightarrow \mathbb{R}$ has the Baire property and $x \in[0,1)$. Let $E_{x, f}^{+}$be equal to

$$
\left\{y \in \mathbb{R}: x \text { is a right-hand } \mathcal{I} \text {-density point of }\left\{t \in(x, 1]: \frac{f(t)-f(x)}{t-x} \leq y\right\}\right\} .
$$

The upper right $\mathcal{I}$-approximate derivative of $f$ at the point $x$ is defined as $\inf E_{x, f}^{+}$and is denoted by $\overline{f_{\mathcal{I}}^{\prime+}}(x)$. If in the definition of $E_{x, f}^{+}$we replace " $\leq$" by " $\geq$ " then the lower right $\mathcal{I}$-approximate derivative of $f$ at $x$ is defined as $\sup E_{x, f}^{+}$and is denoted by $\underline{f_{\mathcal{I}}^{\prime}}(x)$. We adopt the usual convention $\inf \emptyset=+\infty$ and $\sup \emptyset=-\infty$. The lower and the upper left $\mathcal{I}$-approximate derivatives of $f$ at $x \in(0,1]$ are defined analogously. If the lower and upper one-sided 
$\mathcal{I}$-approximate derivatives are equal, their common value is called the one sided $\mathcal{I}$-approximate derivative and is denoted by $f_{\mathcal{I}}^{\prime+}(x)$ or $f_{\mathcal{I}}^{\prime-}(x)$. Finally, $f$ is $\mathcal{I}$-approximately differentiable at $x \in(0,1)$, if $f_{\mathcal{I}}^{\prime+}(x)=f_{\mathcal{I}}^{\prime-}(x) \in \mathbb{R}$ (the common value is written as $\left.f_{\mathcal{I}}^{\prime}(x)\right)$. We additionally put $f_{\mathcal{I}}^{\prime}(0)=f_{\mathcal{I}}^{\prime}(0)$ and $f_{\mathcal{I}}^{\prime}(1)=f_{\mathcal{I}}^{\prime}-(1)$. The definitions given above are analogous to those already well-known from the measure case; e.g. see [G, Def. 16.21], [Br, Chap. 10] and compare with $[\mathrm{LW}]$.

Theorem 2.1. For almost all (in the category sense) functions $f$ in $C[0,1]$, a finite right (respectively, left) $\mathcal{I}$-approximate derivative $f_{\mathcal{I}}^{\prime+}(x)$ (respectively, $\left.f_{\mathcal{I}}^{\prime-}(x)\right)$ can exist for points $x \in[0,1]$ that form a meager set in $[0,1]$.

Proof. First, define a partial function $\Phi$ from $[0,1] \times C[0,1]$ to $\mathbb{R}$ by $\Phi(x, f)=$ $f_{\mathcal{I}}^{\prime}+(x)$. Denote $X=[0,1]$ and $Y=C[0,1]$. Observe that the complement of the domain $D$ of $\Phi$ is equal to $F \cup G \cup H$ where

$F=\left\{(x, f) \in X \times Y: \underline{f_{\mathcal{I}}^{\prime+}}(x)<\overline{f_{\mathcal{I}}^{\prime+}}(x)\right\}$,
$G=\left\{(x, f) \in X \times Y: \overline{f_{\mathcal{I}^{\prime}}}(x)=+\infty\right\}$,
$H=\left\{(x, f) \in X \times Y: \underline{f_{\mathcal{I}}^{\prime}}(x)=-\infty\right\}$.

Observe that, for an arbitrary $w \in \mathbb{R}$, the inequality $\overline{f_{\mathcal{I}}^{\prime+}}(x)>w$ is equivalent to

$$
(\exists n \in \mathbb{N})(\forall y \in \mathbb{R})\left(y \notin E_{x, f}^{+} \vee y>w+1 / n\right) \vee(\forall y \in \mathbb{R})\left(y \notin E_{x, f}^{+}\right)
$$

where $E_{x, f}^{+}$is given by the formula (1). Note that $y \notin E_{x, f}^{+}$if and only if $x$ is not a right-hand $\mathcal{I}$-density point of the set

$$
A_{x, f, y}^{+}:=\left\{t>x: \frac{f(t)-f(x)}{t-x} \leq y\right\}
$$

which, by Lemma 2.1, is equivalent to

$$
\begin{aligned}
&(\exists(a, b) \subset(0,1))(\forall k, m)(\exists n>m)(\forall(c, d) \subset(a, b)) \\
&\left(|d-c|<1 / k \vee(\exists s \in \mathbb{R})\left(s \in((c / n)+x,(d / n)+x) \wedge s \notin A_{x, f, y}^{+}\right)\right)
\end{aligned}
$$

where $a, b, c, d$ are rationals and $k, m, n$-positive integers. If $c, d, n$ are fixed then the formula $(\exists s \in \mathbb{R})\left(s \in((c / n)+x,(d / n)+x) \wedge s \notin A_{x, f, y}^{+}\right)$of the variables $(x, f, y) \in X \times Y \times \mathbb{R}$ describes an open set. Hence the formula 
$y \notin E_{x, f}^{+}$describes a Borel set in $X \times Y \times \mathbb{R}$. Consequently, by (2), the formula $\overline{f_{\mathcal{I}}^{\prime+}}(x)>w$ of the variables $(x, f) \in X \times Y$ describes a coanalytic set in $X \times Y$. (See $[\mathrm{Ke}, 32 \mathrm{~A}]$.$) Hence the set$

$$
F^{w}=\left\{(x, f) \in X \times Y: \overline{f_{\mathcal{I}}^{\prime+}}(x)>w\right\}
$$

possesses the Baire property [Ke, Th. 21.6]. Similarly, one can show that the set

$$
F_{w}=\left\{(x, f) \in X \times Y: \underline{f_{\mathcal{I}}^{\prime}}(x)<w\right\}
$$

has the Baire property. Consequently, $F=\bigcup_{w}\left(F_{w} \cap F^{w}\right)$ possesses the Baire property where the index $w$ runs over all rationals. Let us consider the set $G$. Since $\overline{f_{\mathcal{I}}^{\prime+}}(x)=+\infty$ is equivalent to $E_{x, f}^{+}=\emptyset$, we infer, as above, that $G$ is coanalytic and therefore it possesses the Baire property. It can be shown similarly that $H$ has the Baire property. Consequently, $D$ has the Baire property. Now, observe that the mapping $\Phi: D \rightarrow \mathbb{R}$ has the Baire property since $\Phi(x, f)=\overline{f_{\mathcal{I}}^{\prime+}}(x)$ for $(x, f) \in D$ and we have already proved that the set $\left\{(x, f) \in X \times Y: w<\overline{f_{\mathcal{I}}^{\prime+}}(x)<+\infty\right\}$ has the Baire property for each $w \in \mathbb{R}$.

Next, notice that, for each $x \in X$, the set $D(x)$ is a linear subspace of $Y$ and the mapping $\Phi(x, \cdot)$ is linear. (Compare [G, Th. 16.11]; the respective properties for $\mathcal{I}$-approximate derivatives are analogous.) Finally, note that $\Phi(x, \cdot)$ is discontinuous. Indeed, fix $x \in X$ and put $f_{n}(t)=(1 / n) \sin n(t-x)$ and $f_{0}(t)=0$ for $n \in \mathbb{N}$ and $t \in X$. Then $\left(f_{n}\right)$ tends uniformly to $f_{0}$ (thus $f_{n} \rightarrow f_{0}$ in $Y$ ) but for $x \in X \backslash\{1\}$ we have

$$
\Phi\left(x, f_{n}\right)=f_{n}^{\prime}(x)=\cos 0=1 \rightarrow 1 \neq 0=f_{0}^{\prime}(x)=\Phi\left(x, f_{0}\right) .
$$

Now by Proposition 2.1, we obtain the assertion. The proof for $f_{\mathcal{I}}^{\prime-}$ is analogous.

Acknowledgements. The first author would like to thank Petr Holický and Ludek Zajíček for valuable comments concerning Theorem 1.2. We are indebted to the referee who has pointed out a gap in a former version of the paper.

\section{References}

[B] S. Banach, Über die Baire'sche Kategorie gewisser Funktionenmengen, Studia Math. 3 (1931), 174-179.

[Br] A. Bruckner, Differentiation of Real Functions, (2nd ed.) CRM Monograph Series, vol 5, Amer. Math. Soc., Providence, Rhode Island 1994. 
[CLO] K. Ciesielski, L. Larson, K. Ostaszewski, I-density continuous functions, Mem. Amer. Math. Soc. 107 (515) (1994).

[G] R. A. Gordon, The Integrals of Lebesgue, Denjoy, Perron, and Henstock, Graduate Studies in Math., vol. 4, Amer. Math. Soc., Providence, Rhode Island 1994.

[J] V. Jarnik, Sur les nombres dérivées approximatifs, Fund. Math. 22 (1934), 4-16.

[Jo] S. H. Jones, Applications of the Baire Category theorem, Real Anal. Exchange 23 (1997/8), 363-394.

[Ke] A. S. Kechris, Classical Descriptive Set Theory, Springer-Verlag, New York 1994.

[Kh] A. B. Kharazishvili, Applications of Point Set Theory in Real Analysis, Mathematics and Its Applications, vol. 429, Kluwer Academic Publishers, Dordrecht 1998.

[LW] E. Łazarow, W. Wilczyński, I-approximate derivatives, Radovi Mat. 5 (1989), 15-27.

[M] S. Mazurkiewicz, Sur les fonctions non derivables, Studia Math. 3 (1931), 92-94.

[O] J. C. Oxtoby, Measure and Category, Springer-Verlag, New York 1971.

[W] W. Wilczyński, A category analogue of the density topology, approximate continuity, and the approximate derivative, Real Anal. Exchange 10 (1984-85), 241-265 